\title{
Prognostic significance of $c-$ Myc expression in soft tissue leiomyosarcoma
}

\author{
Athanasios C Tsiatis ${ }^{1}$, Megan E Herceg², Vicki L Keedy ${ }^{3}$, Jennifer L Halpern², \\ Ginger E Holt ${ }^{2}$, Herbert S Schwartz ${ }^{2}$ and Justin MM Cates ${ }^{4}$ \\ ${ }^{1}$ Department of Pathology, Johns Hopkins University, Baltimore, MD, USA; ${ }^{2}$ Department of Orthopaedics and \\ Rehabilitation, Vanderbilt Orthopaedic Institute, Nashville, TN, USA; ${ }^{3}$ Division of Hematology/Oncology, \\ Department of Medicine, Vanderbilt University Medical Center, Nashville, TN, USA and ${ }^{4}$ Department of \\ Pathology, Vanderbilt University Medical Center, Nashville, TN, USA
}

\begin{abstract}
The biological potential of soft tissue leiomyosarcoma is difficult to predict using current standard prognostic parameters, and control of systemic disease is challenging with current chemotherapeutic protocols. Additional prognostic markers and alternative treatment options are very much required. Previous studies implicate upregulation of the oncogenic nuclear transcription factor $c-M y c$ with aggressive behavior of many solid tumors. Therefore, this oncoprotein was evaluated as a prognostic marker for overall and metastasis-free survival in leiomyosarcoma. Immunohistochemical stains for $c$-Myc were performed on 28 cases of leiomyosarcoma occurring in the deep somatic soft tissues. Comparisons of Kaplan-Meier survival curves stratified by $c-M y c$ status and conventional prognostic factors (histological grade, tumor size, and tumor stage) were evaluated using standard univariate statistical methods. A subsequent multivariate survival analysis was carried out according to the Cox proportional hazards regression model adjusting for potential confounding prognostic factors. A total of 15 cases (54\%) were positive for nuclear $c-M y c$ expression. Patients with $c-M y c-$ positive tumors had significantly shorter metastasis-free survival intervals compared with those with $c$-Mycnegative tumors (median, 9 months vs. $>94$ months; $P=0.014)$. $c$-Myc positivity also correlated with decreased overall survival (median, 23 months vs. $>94$ months; $P=0.017$ ). Histological grade was the only other prognostic marker predictive of poor outcome in the univariate analyses. In the multivariate survival analysis, only $c-M y c$ status reached statistical significance, suggesting that it is an important and independent predictor of prognosis in leiomyosarcoma. Detection of nuclear $c-M y c$ in leiomyosarcoma predicts decreased overall and metastasis-free survival, independent of standard prognostic variables, tumor size, histological grade, and TNM stage. The expression of this oncoprotein may represent a useful prognostic marker and potential therapeutic target in leiomyosarcoma.

Modern Pathology (2009) 22, 1432-1438; doi:10.1038/modpathol.2009.113; published online 24 July 2009
\end{abstract}

Keywords: $c-M y c$; leiomyosarcoma; prognosis; immunohistochemistry

Leiomyosarcoma, which is a soft tissue sarcoma demonstrating smooth muscle differentiation, is a tumor prone to local and distant relapse. At present, histological grade, tumor size, and anatomical location are the only pathological factors that are independently predictive of patient outcome. ${ }^{1,2}$ However, significant heterogeneity exists within these risk groups, and patients with tumors deemed

Correspondence: Dr JMM Cates, MD, PhD, Department of Pathology, Medical Center North C-3321, Vanderbilt University Medical Center, 1161 21st Ave South, Nashville, TN 37232, USA. E-mail: justin.m.cates@vanderbilt.edu

Received 26 May 2009; revised 27 June 2009; accepted 29 June 2009; published online 24 July 2009 low to intermediate risk have reported 5-year metastasis rates of approximately 8 and $32 \%$, respectively. ${ }^{1,3}$ Further research into prognostic markers and alternative treatment options is greatly required.

Recently, the downregulation of integrin alpha 7 (ITGA7) gene expression in leiomyosarcoma has been correlated with an increased risk of metastasis and decreased disease-free survival. ${ }^{4,5}$ Integrins are critical mediators of the interactions between cells and the extracellular matrix, and as such are important regulators of cellular differentiation and migration. ITGA7 is a member of the $\alpha$-integrin family, which when heterodimerized with integrin $\beta-1$, is a integral membrane receptor for laminin, a 
major component of the extracellular basement membrane. ${ }^{6}$ Other experimental data have implicated the oncoprotein $C-M y c$ as a direct repressor of ITGA7 gene transcription. ${ }^{7}$

The $c$-Myc gene was originally recognized as a transforming sequence in the avian myelocytomatosis retrovirus and is a member of the Myc gene family that also includes $N-M y c$ and $L-M y c$. The $C$ $M y c$ protein acts as a transcription factor involved in the regulation of numerous critical cell processes, including cell-cycle regulation, cellular differentiation, apoptosis, adhesion, and migration. ${ }^{8,9}$ The currently recognized mechanisms of its oncogenic activation include gene amplification, chromosomal translocation, and insertional mutagenesis. ${ }^{8} C-M y c$ expression has been correlated with poor prognosis in many solid tumors, including osteosarcoma and synovial sarcoma. ${ }^{10-12}$ Overexpression of $c-M y c$ has also been described in high-grade liposarcoma, skeletal chondrosarcoma, and uterine leiomyosarcoma. ${ }^{13-16}$ However, the $c-M y c$ oncoprotein has not been studied extensively in other sarcomas, particularly leiomyosarcoma occurring in the somatic soft tissues. ${ }^{17-19}$

In light of recent data showing that $C$ - $M y c$ downregulates ITGA7 expression, we evaluated whether immunohistochemical staining for $c-M y c$ might serve as a surrogate prognostic marker for decreased ITGA7 expression in leiomyosarcoma. We hypothesized that the overexpression of $c$-Myc would be associated with decreased overall and metastasisfree survival.

\section{Materials and methods}

\section{Study Cases}

This study was approved by the Institutional Review Board at Vanderbilt University; the requirement for informed consent was waived by this committee. The surgical pathology archives at the Vanderbilt University Medical Center (Nashville, TN, USA) were searched for all primary surgical resections of leiomyosarcoma performed between 1994 and 2006. Cases occurring in the subcutaneous soft tissues or involving viscera, including the female genital tract were specifically excluded. In all, 29 cases were retrieved. One low-grade tumor sample recorded as a pelvic mass from a 61-year-old female was excluded because it strongly and diffusely expressed estrogen and progesterone receptors and was considered a smooth muscle neoplasm of gynecological type.

Original hematoxylin and eosin-stained slides were reviewed and blocks were selected for immunohistochemistry. All cases showed classic histopathological features suggestive of leiomyosarcoma (elongate cells with blunt-ended nuclei and relatively abundant eosinophilic cytoplasm forming interweaving fascicles), at least focally. Overall, 17 of the 20 cases tested were positive for desmin expression.
The three desmin-negative tumors showed strong and diffuse staining for $\alpha$-smooth muscle actin. Histological grade was assessed using the French Federation of Cancer Centers Sarcoma Group criteria. ${ }^{20}$ Clinicopathological parameters, including patient age, sex, tumor location, size (greatest linear dimension), and American Joint Committee on Cancer TNM stage (6th edn), were recorded through a retrospective chart review. ${ }^{21}$

\section{c-Myc Immunohistochemistry}

Formalin-fixed, paraffin-embedded tissue sections were pretreated in citrate buffer in a steamer for $25 \mathrm{~min}$ and subsequently incubated with a 1:200 dilution of monoclonal anti- $c-M y c$ antibody (clone 9E10, Sigma-Aldrich, St Louis, MO, USA) for $1 \mathrm{~h}$ at room temperature. Specific antibody binding was detected using an EnVision + kit according to the manufacturer's recommendations (Dako, Carpinteria, CA, USA). A representative section of leiomyosarcoma incubated with diluent alone instead of primary antibody was run in parallel as a negative control. In accordance with previous studies, tumors with specific staining in $>5 \%$ of neoplastic nuclei were scored as positive for $c-M y c$ overexpression. ${ }^{10}$ Slides were independently reviewed by two of the authors (ACT and JMMC) blinded to patient outcome; there were no discrepancies in the recorded $C$-Myc scores.

\section{Statistical Analysis}

The principal study end points were overall survival and metastasis-free survival. Relationships between $c$-Myc expression and other clinicopathological and prognostic variables were also explored. Overall survival was measured as the time elapsed from the date of primary surgical resection to the date of death from any cause and was censored only for patients known to be alive at last contact. Metastasis-free survival was calculated from the date of surgical resection to the date on which radiographic evidence of metastatic disease was first documented and was censored for death and patients without radiographic evidence of metastasis at the last follow-up. Dates of death, last clinical follow-up, or documentation of distant metastasis were obtained by chart review of electronic medical records. Date of patient death was confirmed by searching the Social Security Death Index. ${ }^{22}$ There were no instances of missing data in this study cohort.

Clinicopathological and prognostic parameters were correlated with $C$-Myc status using standard univariate statistical methods (GraphPad Prism v5.01, La Jolla, CA, USA). For ordinal and nonGaussian continuous data (TNM stage, histological grade, and tumor size), the Mann-Whitney test was used. Categorical data were evaluated by Fisher's exact test using a web-based calculator. ${ }^{23}$ Unless 
otherwise stated, two-tailed $P$-values $\leq 0.05$ were considered statistically significant.

Kaplan-Meier survival curves for the different subgroups of prognostic variables were compared using the log-rank (Mantel-Haenszel) test. Survival probabilities for continuous variables were evaluated using both univariate regression analysis and the log-rank test for trend after stratification. Tumor size was stratified into tertiles $(<4.99,5-9.99$, and $>10.0 \mathrm{~cm}$ ) and patient age was approximately stratified into quartiles $(<39,40-59,60-79$, and $\geq 80$ years). The effect of $c-M y c$ status at the time of primary surgical resection of leiomyosarcoma was assessed by multivariable survival analysis according to the Cox proportional hazards regression model, adjusting for potential confounding prognostic factors, such as histological grade, tumor size, and TNM stage. Other clinicopathological parameters associated with the value of $P<0.20$ in univariate analysis were also included in the regression models. Multivariable survival analysis was carried out using the survival package installed on R software (v2.8.1). ${ }^{24,25}$ For multivariate analysis of the effect of AJCC TNM stage on metastasisfree survival, stage IV cases were reclassified as if metastases were not present at the time of diagnosis. No interactions between $c-M y c$ expression and any of the covariables studied were detected when interaction terms were included in the model.

\section{Results}

The cohort consisted of 14 females and 14 males. Neoadjuvant radiation therapy was administered to four patients; one patient received preoperative combination radiation and chemotherapy. The median follow-up for the entire cohort was 23 months (range: 6-94 months) during which time 12 patients (43\%) died and 12 patients (43\%) developed metastatic disease; metastases were detected during initial staging evaluations in 3 other patients. The median overall survival for the entire cohort was 35 months, and the median metastasis-free survival was 23 months. Nuclear immunoreactivity for $c-M y c$ was detected in 15 of 28 tumors (54\%) (Figure 1). The expression of $c-M y c$ did not correlate with prognostic variables, such as histological grade, tumor size, anatomical location, or TNM stage (Table 1). In addition, no significant associations between $c-M y c$ expression and patient age, gender,

Table 1 Comparison of clinicopathological features of $c$-Mycnegative and $c$-Myc-positive soft tissue leiomyosarcoma

\begin{tabular}{|c|c|c|c|}
\hline & $\begin{array}{c}c-M y c- \\
\text { negative } \\
(\mathrm{n}=13)\end{array}$ & $\begin{array}{l}c-M y c- \\
\text { positive } \\
(\mathrm{n}=15)\end{array}$ & $\begin{array}{c}\text { Statistical } \\
\text { significance }^{\mathrm{a}}\end{array}$ \\
\hline Age (years) & $58.9 \pm 4.3$ & $59.3 \pm 4.4$ & $P=0.95$ \\
\hline Sex & & & $P=0.45$ \\
\hline Female & 8 & 6 & \\
\hline Male & 5 & 9 & \\
\hline Anatomical location & & & $P=0.91$ \\
\hline Lower extremity & 8 & 8 & \\
\hline Upper extremity & 3 & 3 & \\
\hline Retroperitoneum & 1 & 1 & \\
\hline Paraspinal & 0 & 2 & \\
\hline Pelvis & 1 & 1 & \\
\hline TNM stage & & & $P=0.26$ \\
\hline Stage I & 1 & 1 & \\
\hline Stage II & 5 & 4 & \\
\hline Stage III & 7 & 7 & \\
\hline Stage IV & 0 & 3 & \\
\hline Histological grade & & & $P=0.87$ \\
\hline High & 9 & 10 & \\
\hline Intermediate & 3 & 3 & \\
\hline Low & 1 & 2 & \\
\hline Tumor size $(\mathrm{cm})$ & $6.2 \pm 0.9$ & $8.8 \pm 1.7$ & $P=0.18$ \\
\hline Radiation therapy & & & $P=0.06$ \\
\hline Neoadjuvant & 0 & 5 & \\
\hline Adjuvant or NA & 10 & 10 & \\
\hline
\end{tabular}

NA, not administered.

${ }^{a}$ Patient age was evaluated using Student's $t$-test.

TNM stage, histological grade, and tumor size were evaluated using the Mann-Whitney test.

Other categories were compared using Fisher's exact test. a

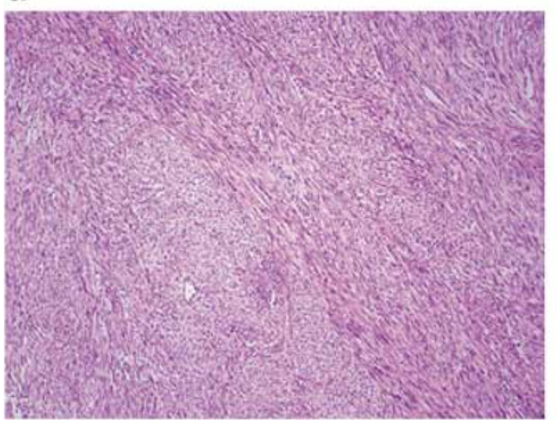

b

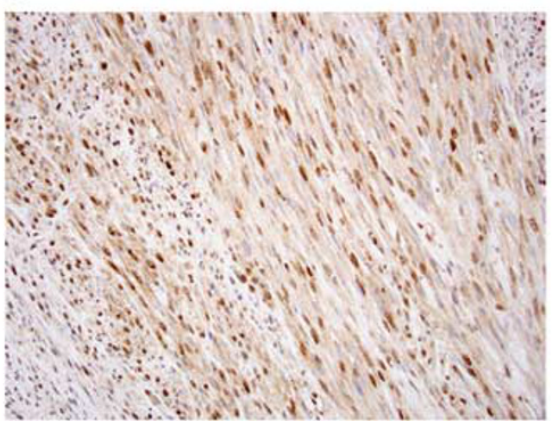

C

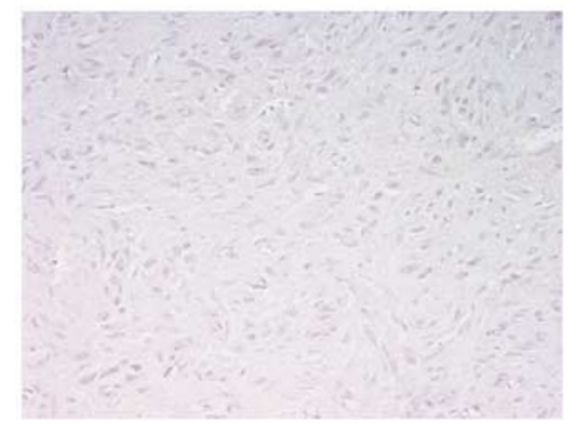

Figure 1 Immunohistochemical stains for $c-M y c$ in soft tissue leiomyosarcoma (a, H\&E) representative of positive (b) and negative (c) cases (original magnification, $\times 200$ ). 
Table 2 Univariate and multivariate metastasis-free survival analysis

\begin{tabular}{|c|c|c|c|}
\hline Parameter & $H R$ & $95 \% C I$ & P-value \\
\hline \multicolumn{4}{|l|}{ Univariate } \\
\hline$C-M y c$ (positive vs negative) & 3.7 & $1.30-10.49$ & 0.01 \\
\hline Neoadjuvant XRT vs NA & 17.82 & $2.50-127.1$ & $<0.01$ \\
\hline Histological grade (grades 1-2 vs grade 3 ) & 0.28 & $0.09-0.90$ & 0.03 \\
\hline Tumor size ${ }^{\text {a }}$ (first tertile vs third tertile) & 0.2 & $0.04-0.94$ & 0.07 \\
\hline TNM stage (stages I-II vs stage III) & 0.41 & $0.13-1.30$ & 0.13 \\
\hline Patient age $^{\mathrm{a}}(<60$ vs $\geq 60$ years $)$ & 0.81 & $0.29-2.25$ & 0.43 \\
\hline Sex (female vs male) & 0.5 & $0.16-1.61$ & 0.25 \\
\hline Anatomical site (extremity vs non-extremity) & 0.68 & $0.18-2.61$ & 0.58 \\
\hline \multicolumn{4}{|l|}{ Multivariate } \\
\hline$c-M y c$ (positive vs negative) & 3.56 & $1.04-12.34$ & 0.04 \\
\hline Neoadjuvant XRT vs NA & 0.77 & $0.16-3.73$ & 0.75 \\
\hline Histological grade & 2.81 & $0.88-9.01$ & 0.08 \\
\hline Tumor size & 1.15 & $0.98-1.35$ & 0.1 \\
\hline TNM stage ${ }^{\mathrm{b}}$ & 0.82 & $0.23-2.91$ & 0.76 \\
\hline
\end{tabular}

CI, confidence interval; HR, hazard ratio; NA, not administered; XRT, radiation therapy.

${ }^{\mathrm{a}}$ See 'Statistical Analysis' subsection under the section 'Material and methods' for details regarding stratifications.

${ }^{b}$ For multivariate analysis of the effect of TNM stage on metastasis-free survival, the 3 -stage IV cases were reclassified as if metastases were not present at diagnosis.
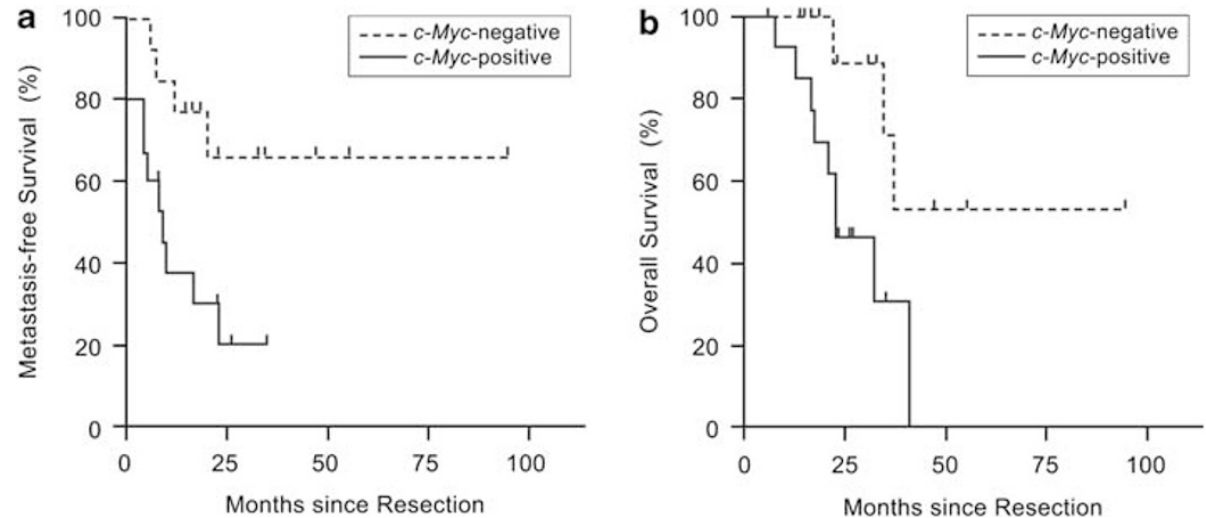

Figure 2 Metastasis-free (a) and overall (b) Kaplan-Meier survival curves of patients with $c$-Myc-positive (-) and $c$-Myc-negative (----) soft tissue leiomyosarcoma.

or exposure to neoadjuvant radiation therapy were observed.

Log-rank tests performed on Kaplan-Meier survival curves disclosed significantly decreased metastasis-free survival for patients with $c$-Myc-positive leiomyosarcoma compared with $c-M y c$-negative tumors (median, 9 months vs. $>94$ months; $P=0.014$ ) (Table 2 and Figure 2a). Increased risk of metastasis was also noted for patients with highgrade (grade 3) leiomyosarcoma and for those patients who received preoperative radiation therapy. Positive $c-M y c$ status was the only variable that reached statistical significance for decreased metastasis-free survival in subsequent Cox proportional hazards regression analysis (Table 2).

Overall survival intervals were also significantly shorter in patients with $c-M y c$-positive leiomyosarcoma (median, 23 months vs. >94 months; $P=0.017$ ) (Table 3 and Figure $2 b$ ). None of the other parameters that were studied showed a significant effect on overall survival in univariate analysis, although a trend was noted for histological grade. Despite a relatively wide $95 \%$ confidence interval, $c-M y c$ status was the only factor independently associated with increased risk of death in multivariate regression analysis (Table 3 ).

Of the three patients with leiomyosarcoma of low histological grade (grade 1), two were positive for $c-M y c$ expression. One of the $c-M y c$-positive cases presented with metastatic disease and died 13 months after surgical resection of the primary tumor; the other is alive without evidence of the disease 8 months after surgical resection. The one patient with a $c-M y c$-negative, low-grade leiomyosarcoma is an event-free survivor at 23 months of clinical follow-up. The three patients in whom metastatic disease was discovered during initial staging studies were all positive for $c-M y c$, 
Table 3 Univariate and multivariate overall survival analysis

\begin{tabular}{|c|c|c|c|}
\hline Parameter & $H R$ & $95 \% C I$ & $P$-value \\
\hline \multicolumn{4}{|l|}{ Univariate } \\
\hline$c-M y c$ (positive vs negative) & 4.2 & $1.29-13.61$ & 0.02 \\
\hline Neoadjuvant XRT vs NA & 2.86 & $0.65-12.54$ & 0.16 \\
\hline Histological grade (grades $1-2$ vs grade 3 ) & 0.32 & $0.10-1.06$ & 0.06 \\
\hline Tumor size ${ }^{\text {a }}$ (first tertile vs third tertile) & 0.51 & $0.14-1.91$ & 0.36 \\
\hline TNM stage (stages I-II vs stages III-IV) & 0.72 & $0.02-2.28$ & 0.57 \\
\hline Patient age $^{\mathrm{a}}(<60$ vs $\geq 60$ years $)$ & 1.03 & $0.33-3.26$ & 0.92 \\
\hline Sex (female vs male) & 1.22 & $0.39-3.83$ & 0.73 \\
\hline Anatomical site (extremity vs non-extremity) & 0.7 & $0.16-2.97$ & 0.62 \\
\hline \multicolumn{4}{|l|}{ Multivariate } \\
\hline$C-M y c$ (positive vs negative) & 5.6 & $1.00-31.46$ & 0.05 \\
\hline Neoadjuvant XRT vs NA & 0.89 & $0.10-7.77$ & 0.92 \\
\hline Histological grade & 4.14 & $0.81-21.27$ & 0.09 \\
\hline TNM stage & 1.61 & $0.59-4.41$ & 0.36 \\
\hline Tumor size & 1.02 & $0.85-1.22$ & 0.85 \\
\hline
\end{tabular}

CI, confidence interval; HR, hazard ratio; NA, not administered; XRT, radiation therapy.

${ }^{\text {a }}$ See 'Statistical Analysis' subsection under the section 'Material and methods' for details regarding stratifications.

and all three died within 23 months of surgical resection.

\section{Discussion}

We found that $c-M y c$ expression significantly correlates with both metastasis-free and overall survival in patients with resected leiomyosarcoma. Multivariate survival analysis showed that $c-M y c$ status was the only prognostic factor that independently predicted decreased survival intervals. Although histological grade and pathological stage are widely accepted prognostic factors for leiomyosarcoma, it remains very difficult to predict outcomes for individual patients stratified using these parameters alone. ${ }^{2}$ Recently, it has been shown that ITGA7 gene expression is downregulated 40 -fold in leiomyosarcoma with high metastatic potential and is associated with decreased metastasis-free survival., ${ }^{4,5}$ The lack of a commercially available antibody against ITGA7 for use on formalin-fixed, paraffinembedded tissue prompted us to evaluate upstream regulators of ITGA7 as potential molecular prognostic markers for leiomyosarcoma.

$c-M y c$ downregulates ITGA7 gene transcription by binding to a double E-box in the ITGA7 promoter region. ${ }^{7}$ Therefore, we examined whether the detection of nuclear $c-M y c$ was prognostically significant in a series of leiomyosarcoma. Nuclear $c-M y c$ expression did not correlate with other accepted prognostic markers for leiomyosarcoma. However, $c-M y c$ expression was associated with decreased metastasis-free and overall survival, consistent with previous experimental data that showed poor outcomes in patients with leiomyosarcoma with decreased ITGA7 expression.

In $\mathrm{C} 2 \mathrm{C} 12$ myoblasts, overexpression of ITGA7 is associated with increased binding of laminin and decreased binding of fibronectin. ${ }^{26,27}$ Conversely, the downregulation of ITGA7 modulates interactions between integrins and the extracellular matrix such that fibronectin binding is favored. ${ }^{27}$ In mammary epithelial cells, fibronectin-dependent signaling has been shown to upregulate $C-M y c$ expression through the activation of the MEK-ERK pathway. ${ }^{28}$ Although not yet established, it is possible that the $C$-Mycmediated inhibition of ITGA7 increases fibronectin binding and represents a positive feedback loop that stimulates $C$-Myc overexpression in a growth factorindependent manner. The potential significance of this pathway is shown by evidence that the MEK-ERK inhibitor U0126 decreases proliferation of rhabdomyosarcoma cells concomitant with decreased intracellular levels of $c-M y c{ }^{29,30}$ Perhaps the $c-M y c$ status of leiomyosarcoma might identify those tumors most susceptible to MEK-ERK inhibition.

The consequences of $c-M y c$ overexpression are pleiotropic and include dysregulation of the cell cycle, suppression of apoptosis, and cellular transformation, in addition to inhibition of ITGA7 and subsequent alteration of cell adhesion and migratory properties. ${ }^{8,9}$ Studies directly targeting $c-M y c$ expression in transgenic mouse models have shown profound anti-neoplastic effects, including potentiation of chemotherapeutic agents, suggesting that that other therapeutic strategies may improve survival in patients with $C$-Myc-positive leiomyosarcoma. ${ }^{12,31-34}$ Strategies to inhibit transcription or translation of $c-M y c$ mRNA that are currently under investigation or in development include the use of antisense oligonucleotides, triple helix-forming oligonucleotides, cationic porphyrins, and phosphorodiamidate morpholino oligomers (AVI-5126, ClinicalTrials.gov identifier: NCT00777842). ${ }^{35}$ Together with the results of this study, these advances should prompt further investigation into current therapeutic approaches to soft tissue leiomyosarcoma. ${ }^{36}$ 
In summary, our data suggest that immunohistochemical detection of $c-M y c$ may be useful in predicting increased risk of metastasis and shorter overall survival in patients with leiomyosarcoma. Such ancillary information may assist in clinical decision making regarding the intensity and type of adjuvant therapy administered. Consideration of larger, multi-institutional studies is recommended to validate these data. Given the adverse prognosis that $c-M y c$ overexpressing tumors confer on patients and other preclinical data collected thus far, clinical trials evaluating the role of anti-c-Myc or anti-MEK-ERK pharmacotherapeutic agents in treating sarcomas such as leiomyosarcoma should also be considered.

\section{Acknowledgement}

The authors thank Sandy Olson, Lesley Albert, and Jean McClure for their technical and editorial assistance.

\section{Disclosure/conflict of interest}

Vicki L Keedy, MD, receives commercial research support for ongoing clinical trials in collaboration with the following agencies: Ariad Pharmaceuticals, Ziopharm Oncology, Inc., and Amgen Oncology Therapeutics.

\section{References}

1 Farshid G, Pradhan M, Goldblum J, et al. Leiomyosarcoma of somatic soft tissues: a tumor of vascular origin with multivariate analysis of outcome in 42 cases. Am J Surg Pathol 2002;26:14-24.

2 Svarvar C, Bohling T, Berlin O, et al. Clinical course of nonvisceral soft tissue leiomyosarcoma in 225 patients from the Scandinavian Sarcoma Group. Cancer 2007; 109:282-291.

3 Coindre JM, Terrier P, Bui NB, et al. Prognostic factors in adult patients with locally controlled soft tissue sarcoma. A study of 546 patients from the French Federation of Cancer Centers Sarcoma Group. J Clin Oncol 1996;14:869-877.

4 Ren B, Yu YP, Jing L, et al. Gene expression analysis of human soft tissue leiomyosarcomas. Hum Pathol 2003;34:549-558.

5 Ren B, Yu YP, Tseng GC, et al. Analysis of integrin alpha7 mutations in prostate cancer, liver cancer, glioblastoma multiforme, and leiomyosarcoma. J Natl Cancer Inst 2007;99:868-880.

6 Belkin AM, Stepp MA. Integrins as receptors for laminins. Microsc Res Tech 2000;51:280-301.

7 Xiao J, Jethanandani P, Ziober BL, et al. Regulation of alpha7 integrin expression during muscle differentiation. J Biol Chem 2003;278:49780-49788.

8 Knoepfler PS. Myc goes global: new tricks for an old oncogene. Cancer Res 2007;67:5061-5063.

9 Pelengaris S, Khan M, Evan G. c-MYC: more than just a matter of life and death. Nat Rev Cancer 2002;2: 764-776.
10 Shen J, Scotlandi K, Baldini N, et al. Prognostic significance of nuclear accumulation of c-myc and mdm2 proteins in synovial sarcoma of the extremities. Oncology 2000;58:253-260.

11 Gamberi G, Benassi MS, Bohling T, et al. C-myc and c-fos in human osteosarcoma: prognostic value of mRNA and protein expression. Oncology 1998;55: $556-563$.

12 Scionti I, Michelacci F, Pasello M, et al. Clinical impact of the methotrexate resistance-associated genes c-myc and dihydrofolate reductase (DHFR) in highgrade osteosarcoma. Ann Oncol 2008;19:1500-1508.

13 Morrison C, Radmacher M, Mohammed N, et al. Myc amplification and polysomy 8 in chondrosarcoma: array comparative genomic hybridization, fluorescent in situ hybridization, and association with outcome. J Clin Oncol 2005;23:9369-9376.

14 Schneider-Stock R, Boltze C, Jager V, et al. Elevated telomerase activity, c-MYC-, and hTERT mRNA expression: association with tumour progression in malignant lipomatous tumours. J Pathol 2003;199: 517-525.

15 Fotiou SK, Tserkezoglou AJ, Mahera H, et al. Chromosome aberrations and expression of ras and myc oncogenes in leiomyomas and a leiomyosarcoma of the uterus. Eur J Gynaecol Oncol 1992;13:340-345.

16 Jeffers MD, Richmond JA, Macaulay EM. Overexpression of the c-myc proto-oncogene occurs frequently in uterine sarcomas. Mod Pathol 1995;8:701-704.

17 Barrios C, Castresana JS, Kreicbergs A. Clinicopathologic correlations and short-term prognosis in musculoskeletal sarcoma with c-myc oncogene amplification. Am J Clin Oncol 1994;17:273-276.

18 Maillet MW, Robinson RA, Burgart LJ. Genomic alterations in sarcomas: a histologic correlative study with use of oncogene panels. Mod Pathol 1992;5: 410-414.

19 Shin DM, Gupta V, Donner L, et al. Aberrant oncogene expression in uncultured human sarcoma and melanoma. Anticancer Res 1987;7:1117-1123.

20 Kempson RL, Fletcher CDM, Evans HL, et al. Vol 30 3rd series. Armed Forces Institute of Pathology: Washington, DC, 2001, 507pp.

21 Greene FL, Page DL, Fleming ID, et al. (eds) AJCC Cancer Staging Manual 6th edn Springer Verlag: New York, 2002, 421pp.

22 Social security death index. http://www.genesearch. com/socialsecurity.htmlAccessed: 17 February, 2009.

23 Kirkman TW. Statistics to use. http://www.physics. csbsju.edu/stats/ 1996.

24 Therneau T, Lumley L. Survival: survival analysis, including penalised likelihood $\mathrm{R}$ package version $2.34-1$ 2008.

25 R Development Core Team. R: A Language and Environment for Statistical Computing. R Foundation for Statistical Computing: Vienna, 2008; http://www. R-project.org.

26 Liu J, Burkin DJ, Kaufman SJ. Increasing alpha 7 beta 1-integrin promotes muscle cell proliferation, adhesion, and resistance to apoptosis without changing gene expression. Am J Physiol Cell Physiol 2008;294: C627-C640.

27 Nawrotzki R, Willem M, Miosge N, et al. Defective integrin switch and matrix composition at alpha 7-deficient myotendinous junctions precede the onset of muscular dystrophy in mice. Hum Mol Genet 2003;12:483-495. 
28 Benaud CM, Dickson RB. Regulation of the expression of c-Myc by beta1 integrins in epithelial cells. Oncogene 2001;20:759-768.

29 Marampon F, Bossi G, Ciccarelli C, et al. MEK/ERK inhibitor U0126 affects in vitro and in vivo growth of embryonal rhabdomyosarcoma. Mol Cancer Ther 2009;8:543-551.

30 Marampon F, Ciccarelli C, Zani BM. Down-regulation of c-Myc following MEK/ERK inhibition halts the expression of malignant phenotype in rhabdomyosarcoma and in non muscle-derived human tumors. Mol Cancer 2006;5:31.

31 Ponzielli R, Katz S, Barsyte-Lovejoy D, et al. Cancer therapeutics: targeting the dark side of Myc. Eur J Cancer 2005;41:2485-2501.
32 Jain M, Arvanitis C, Chu K, et al. Sustained loss of a neoplastic phenotype by brief inactivation of MYC. Science 2002;297:102-104.

33 Jonkers J, Berns A. Oncogene addiction: sometimes a temporary slavery. Cancer Cell 2004;6:535-538.

34 Xie XK, Yang DS, Ye ZM, et al. Recombinant antisense C-myc adenovirus increase in vitro sensitivity of osteosarcoma MG-63 cells to cisplatin. Cancer Invest 2006;24:1-8.

35 de Nigris F, Balestrieri ML, Napoli C. Targeting c-Myc, Ras and IGF cascade to treat cancer and vascular disorders. Cell Cycle 2006;5:1621-1628.

36 Vita M, Henriksson M. The Myc oncoprotein as a therapeutic target for human cancer. Semin Cancer Biol 2006;16:318-330. 